\title{
Robotic Nipple-Sparing Mastectomy: The Next Step in the Evolution of Minimally Invasive Breast Surgery
}

\author{
Jesse C. Selber, MD, MPH, FACS \\ Department of Plastic Surgery, The University of Texas, MD Anderson Cancer Center, Houston
}

Mastectomy has come a long way since its introduction by Halstead at the turn of the twentieth century. What was once a disfiguring operation guided by a scorched-earth approach has evolved into an elegant dissection, often sparing the nipple-areolar complex, through a small incision hidden in the curvature of the breast. Nipple-sparing mastectomy (NSM) has simultaneously decreased morbidity and increased patient satisfaction and cosmetic results without compromising oncologic outcomes. ${ }^{1-4}$

Despite the clear benefits of NSM, technical challenges of dissecting and removing large specimens through limited incisions have restricted the percentage of qualified candidates that receive NSM. In addition, difficulty visualizing regions of the breast remote from the incision can compromise the oncologic effectiveness of NSM. Robotic nipple-sparing mastectomy (R-NSM) is a natural step in the evolution of minimally invasive breast surgery that has the potential to mitigate the challenges associated with traditional NSM.

In NSM, the upper pole of the breast is the most difficult to access through an inframammary incision. In R-NSM, because of the enlarged optical space created as dissection progresses across the breast, dissection actually becomes easier as one gets further from the access incision. This is the exact opposite scenario from a traditional NSM, where the dissection becomes increasingly difficult, requiring deep retraction, headlights, and awkward positioning to see past the breast tissue into the deep recesses of the breast. In R-NSM, insufflation creates a broad dome of uniform

(C) Society of Surgical Oncology 2018

First Received: 19 July 2018; Published Online: 12 November 2018

J. C. Selber, MD, MPH, FACS

e-mail: JCSelber@mdanderson.org retraction across the horizon line of the dissection, eliminating the need for retraction and maintaining a consistent working distance and line of sight.

Although it has yet to make landfall in the USA, R-NSM was first described by Toesca in 2015 and is catching on in Europe, where early results have been very promising. ${ }^{5} \mathrm{~A}$ low rate of conversion to open mastectomy, low rates of skin necrosis, and high degrees of patient satisfaction characterize early European experience. ${ }^{6,7}$ The manuscript by Lai et al. presents a series in which R-NSM was performed successfully in 23 breasts with immediate breast reconstruction. There was no nipple necrosis and no positive margins, and all patients were satisfied with the procedure. I would like to discuss some general aspects of R-NSM, highlight some points made in this manuscript, and mention a few areas where I agree, and respectfully disagree with the authors' approach.

It will be valuable to highlight a couple of points about this manuscript. First, the authors have chosen to place the incision in the axilla. When performing the robotic dissection, the arms of the DaVinci robot (Intuitive Surgical, Sunnyvale, CA) must drop below the plane of the bed to ascend the dome of the breast. To avoid conflicts with the shoulder and/or injury to the upper arm, it is advantageous to move out of the axilla and onto the lateral chest wall. I have found that an incision lateral to the breast at the level of the NAC, with arms abducted or resting comfortably above the head, provides the best access and least conflict with the patient.

The authors demonstrate ingenuity in overcoming an important technical challenge through use of the Glove Port. Because the remote center of the instruments (the fulcrum around which the instruments rotate) is typically located at the port site, range of motion can be limited close to the incision. By using the Glove Port, the remote center is moved away from the body, permitting full range of motion close to the incision. We use a GelPOINT (Applied 
Medical, Rancho Santa Margarita, CA) for this purpose, but the same effect can be achieved both and probably other ways, as well.

In my opinion, the incision of $3.5 \mathrm{~cm}$ used by the authors is somewhat small for the average breast. The incision must be large enough to remove the intact mastectomy specimen and perform immediate breast reconstruction. A breast larger than an A cup will likely be difficult to remove intact through a $3.5-\mathrm{cm}$ incision. Additionally, in this study, reconstruction was performed with a small, subpectoral implant without use of acellular dermal matrix (ADM). Because of decreased pain, morbidity, elimination of animation deformity, and improved breast esthetics, our standard has become prepectoral breast reconstruction with ADM. It is somewhat difficult to accomplish this procedure through a $3.5-\mathrm{cm}$ incision, so the combination of mastectomy specimen size and breast reconstruction technique has led us to adopt an incision of $4-4.5 \mathrm{~cm}$ for R-NSM.

Although the mean operative time in this study was $118 \mathrm{~min}$, the time dropped to $100 \mathrm{~min}$ towards the end of enrollment, and docking time decreased from 20 to $7 \mathrm{~min}$, appropriate times for R-NSM. Inclusion criteria of earlystage breast cancer only and exclusion of large, ptotic breasts was a good choice for this pilot study. Although high costs were cited as a limitation of the procedure, we have not had any difficulties with reimbursement for robotic breast surgery or reconstruction. The patient satisfaction scale used in this study is not, to my knowledge, validated. For instance the Breast-Q might have been a better choice; however, high scores are noted, and this is both encouraging and expected.

As a matter of technical work flow, the authors of this paper perform the superficial dissection first. I recommend performing the deep dissection off the chest wall first, as this assists with identifying the end-point of the superficial dissection as it opens up into the deep dissection space. I do not doubt that an adequate dissection can be performed in either order. Interestingly, the authors use blue dye delivered with a hypodermic needle at the boundaries of the breast to guide the robotic dissection, as it is easy to become disoriented inside the breast and overdissect the natural boundaries. I also use this approach, and it is confirming to see that it seemed logical and useful to other surgeons, as well. Finally, 7 months is not enough time to assess the oncologic safety of this procedure, although no locoregional recurrence at this time is certainly an excellent beginning.

These are indeed exciting times for robotic breast surgery. I commend the authors of this manuscript for engaging in the cutting edge of this burgeoning field. Mastectomy has come a long way, and R-NSM promises to reduce morbidity and improve cosmetic outcomes even further. Synergies between robotic oncologic and reconstructive surgery will further magnify this effect, reducing the negative impact of an already devastating diagnosis of breast cancer can have on a woman.

\section{REFERENCES}

1. Gerber B, Krause A, Dieterich M, Kundt G, Reimer T. The oncological safety of skin sparing mastectomy with conservation of the nipple-areola complex and autologous reconstruction: an extended follow-up study. Ann Surg. 2009;249(3):461-8.

2. Orzalesi L, Casella D, Santi C, et al. Nipple sparing mastectomy: surgical and oncological outcomes from a national multicentric registry with 913 patients (1006 cases) over a six year period. Breast. 2016;25:75-81.

3. De La Cruz L, Moody AM, Tappy EE, Blankenship SA, Hecht EM. Overall survival, disease-free survival, local recurrence, and nipple-areolar recurrence in the setting of nipple-sparing mastectomy: a meta-analysis and systematic review. Ann Surg Oncol. 2015;22(10):3241-9.

4. Satteson ES, Brown BJ, Nahabedian MY. Nipple-areolar complex reconstruction and patient satisfaction: a systematic review and meta-analysis. Gland Surg. 2017;6(1): 4-13.

5. Toesca A, Peradze N, Galimberti V, Manconi A, Intra M, Gentilini O, Sances D, Negri D, Veronesi G, Rietjens M, Zurrida S, Luini A, Veronesi U, Veronesi P. Robotic nipple-sparing mastectomy and immediate breast reconstruction with implant: first report of surgical technique. Ann Surg. 2015;266:e28-e30.

6. Sarfati B, Honart JF, Leymarie N, Kolb F, Rimareix F. Roboticassisted nipple sparing mastectomy: a feasibility study on cadaveric models. J Plast Reconstr Aesthet Surg. 2016;69(11):1571-2.

7. Toesca A, Peradze N, Manconi A, Galimberti V, Intra M, Colleoni $\mathrm{M}$, et al. Robotic nipple-sparing mastectomy for the treatment of breast cancer: feasibility and safety study. Breast. 2017;31:51-6. 